\title{
A New Iterative Scheme for Non-expansive and Monotone Lipschitz Continuous Mappings
}

\author{
Renu Chugh \\ Department of Mathematics, \\ Maharshi Dayanand University, \\ Rohtak (India)- 124001.
}

\author{
Rekha Rani \\ Department of Mathematics, \\ Maharshi Dayanand University, \\ Rohtak (India)- 124001.
}

\begin{abstract}
The aim of paper is to prove a weak convergenceresult for finding a common of the set of fixed points of a nonexpansive mapping and the set of solutions of a variational inequality problem for a monotone, Lipschitz continuous mapping. Using an example in $\mathrm{C}++$, validity of the result will be proved. Also, we shall find a common element of the set of fixed points of a nonexpansive mapping and the set of zeros of a monotone, Lipschitz continuous mapping.
\end{abstract}

\section{KeyWords}

Fixed Points, Hilbert Spaces, Monotone Mappings, Nonexpansive Mappings, Variational Inequalities.2000 Mathematics Subject Classification: Primary 47H05, 47J05, 47J25

\section{INTRODUCTION}

Let $\mathrm{H}$ be a real Hilbert space with inner product $\langle.,$.$\rangle and$ norm $\|$.$\| , respectively. Let \mathrm{C}$ be a closed convex subset of $\mathrm{H}$. The variational inequality problem is to find $\mathrm{u} \in \mathrm{C}$ such that $<$ $\mathrm{Au}, \mathrm{v}-\mathrm{u}>\geq 0, \quad \forall \mathrm{v} \in \mathrm{C}$.

The set of solutions of variational inequality problem VI(C, A) is denoted by $\Omega$. The variational inequality problem has been extensively studied in literature, see, for example,[1, 2, $5,10]$ and references therein.

Definitions. Let A: $\mathrm{C} \rightarrow \mathrm{H}$ be a mapping of $\mathrm{C}$ into $\mathrm{H}$. A is called monotone if

$<\mathrm{Au}-\mathrm{Av}, \mathrm{u}-\mathrm{v}>\geq 0 \quad \forall \mathrm{u}, \mathrm{v} \in \mathrm{C}$.

A is called $\alpha$-inverse-strongly-monotone $[1,5,9]$ if there exists a positive real number $\alpha$ such that

$<\mathrm{Au}-\mathrm{Av}, \mathrm{u}-\mathrm{v}>\geq \alpha\|\mathrm{Au}-\mathrm{Av}\|^{2} \quad \forall \mathrm{u}, \mathrm{v} \in \mathrm{C}$.

It is easy to see that an $\alpha$-inverse-strongly mapping $\mathrm{A}$ is monotone and Lipschitz continuous but converse is not true. A mapping $\mathrm{S}: \mathrm{C} \rightarrow \mathrm{C}$ is called non-expansive [10-11] if

$\|\mathrm{Su}-\mathrm{Sv}\| \leq\|\mathrm{u}-\mathrm{v}\| \quad \forall \mathrm{u}, \mathrm{v} \in \mathrm{C}$.

We denote by $\mathrm{F}(\mathrm{S})$ the set of fixed points of $\mathrm{S}$. A mapping $\mathrm{S}$ : $\mathrm{C} \rightarrow \mathrm{C}$ is called Lipschitz continuous if there exists a real number $\mathrm{L}>0$ such that $\|\mathrm{Su}-\mathrm{Sv}\| \leq \mathrm{L}\|\mathrm{u}-\mathrm{v}\| \quad \forall$ $\mathrm{u}, \mathrm{v} \in \mathrm{C}$.

Takahashi and Toyoda [12] introduced the following iterative scheme for finding a common element of the set of fixed points of a nonexpansive mapping and the set of solutions of the variational inequality problem for an $\alpha$-inverse-stronglymonotone mapping in a real Hilbert space.

Theorem 1.1. [12] Let $\mathrm{C}$ be a closed convex subset of a real Hilbert space $\mathrm{H}$. Let $\mathrm{A}$ be an $\alpha$-inverse-strongly-monotone mapping of $\mathrm{C}$ into $\mathrm{H}$ and let $\mathrm{S}$ be a nonexpansive mapping of $C$ into itself such that $F(S) \cap \operatorname{VI}(C, A) \neq \phi$. Let $\left\{x_{n}\right\}$ be a sequence generated by $x_{0}=x \in C, \quad x_{n+1}=\alpha_{n} x_{n}+\left(1-\alpha_{n}\right) S$
$\mathrm{P}_{\mathrm{C}}\left(\mathrm{x}_{\mathrm{n}}-\lambda_{\mathrm{n}} A \mathrm{x}_{\mathrm{n}}\right), \quad(1.1)$ for every $\mathrm{n}=0,1,2, \ldots \ldots \ldots$ where $\left\{\lambda_{\mathrm{n}}\right\}$ $\subset[a, b]$ for some a,b $\in(0,2 \alpha)$ and $\left\{\alpha_{n}\right\} \subset[c, d]$ for some $c, d \epsilon$ $(0,1)$. Then, the sequence $\left\{x_{n}\right\}$ converges weakly to some point $z \in F(S) \cap \operatorname{VI}(C, A)$, where, $z=\lim _{n \rightarrow \infty} P_{F(S) \cap V I(C, A)} x_{n}$. Further liduka and Takahashi [4], introduced an iterative scheme to find a common element of the set of fixed points of a nonexpansive mapping and the set of solutions of the variational inequality for an inverse-strongly-monotone mapping in a Hilbert space.

Theorem 1.2. [4] Let $\mathrm{C}$ be a closed convex subset of a real Hilbert space $\mathrm{H}$. Let $\mathrm{A}$ be an $\alpha$-inverse-strongly-monotone mapping of $\mathrm{C}$ into $\mathrm{H}$ and let $\mathrm{S}$ be a nonexpansive mapping of $\mathrm{C}$ into itself such that $\mathrm{F}(\mathrm{S}) \cap \mathrm{VI}(\mathrm{C}, \mathrm{A}) \neq \phi$. Suppose $\mathrm{x}_{1}=\mathrm{x} \epsilon$ $\mathrm{C}$ and let $\left\{\mathrm{x}_{\mathrm{n}}\right\}$ be a sequence generated by

$x_{n+1}=P_{C}\left(\alpha_{n} x+\left(1-\alpha_{n}\right) \operatorname{SP}_{C}\left(x_{n}-\lambda_{n} A x_{n}\right)\right)$,

for every $n=1,2, \ldots \ldots \ldots$ where $\left\{\alpha_{n}\right\}$ is a sequence in $[0,1)$ and $\left\{\lambda_{n}\right\}$ is a sequence in $[0,2 \alpha]$. If $\left\{\alpha_{n}\right\}$ and $\left\{\lambda_{n}\right\}$ are chosen so that $\left\{\lambda_{n}\right\} \subset[a, b]$ for some a, b with $0<\mathrm{a}<\mathrm{b}<2 \alpha$,

$\lim _{n \rightarrow \infty} \alpha_{n}=0, \quad \sum_{n=1}^{\infty} \alpha_{n}=\infty \quad \sum_{n=1}^{\infty}\left|\alpha_{n+1}-\alpha_{n}\right|<\infty$

$\sum_{n=1}^{\infty}\left|\lambda_{n+1}-\lambda_{n}\right|<\infty$

Then $\left\{x_{n}\right\}$ converges strongly to $\mathrm{P}_{\mathrm{F}(\mathrm{S}) \cap \mathrm{VI}(\mathrm{C}, \mathrm{A}) \mathrm{X}}$.

Motivated by above results, we shall prove the weak convergence theorem for finding a common element of the set of fixed points of a nonexpansive mapping and the set of solutions of the variational inequality problem for a monotone and Lipschitz continuous mapping in a real Hilbert space. Also we shall give a numerical example to show the existence of solution. Further, we consider the problem of finding a common element of the set of fixed points of a nonexpansive mapping and the set of zeros of a monotone and Lipschitz continuous mapping.

\section{PRELIMINARIES}

Let $\mathrm{H}$ be a real Hilbert space with inner product $\langle.$, . $\rangle$ and norm $\|$.$\| . Let \mathrm{C}$ be a closed convex subset of $\mathrm{H}$. We shall write $x_{n} \rightarrow x$ to indicate that the sequence $\left\{x_{n}\right\}$ converges weakly to $\mathrm{x} . \mathrm{x}_{\mathrm{n}} \rightarrow \mathrm{x}$ implies that $\left\{\mathrm{x}_{\mathrm{n}}\right\}$ converges strongly to $\mathrm{x}$. It is well known that for any $\mathrm{x} \in \mathrm{H}$, there exists a unique nearest point in $\mathrm{C}$, such that

$\left\|\mathrm{u}-\mathrm{P}_{\mathrm{C}} \mathrm{x}\right\|=\inf \{\|\mathrm{u}-\mathrm{y}\|: \mathrm{y} \in \mathrm{C}\}$

$\mathrm{P}_{\mathrm{C}}$ is called the metric projection of $\mathrm{H}$ onto $\mathrm{C}$.

$\mathrm{P}_{\mathrm{C}}$ is characterized by the properties:

$\mathrm{P}_{\mathrm{C}} \in \mathrm{C}$,

$<\mathrm{x}-\mathrm{P}_{\mathrm{C}} \mathrm{x}, \mathrm{y}-\mathrm{P}_{\mathrm{C}} \mathrm{x}>\leq 0$, for all $\mathrm{x} \in \mathrm{H}, \mathrm{y} \in \mathrm{C}$, 
$\|\mathrm{x}-\mathrm{y}\|^{2} \geq\left\|\mathrm{x}-\mathrm{P}_{\mathrm{C}} \mathrm{x}\right\|^{2}+\left\|\mathrm{y}-\mathrm{P}_{\mathrm{C}} \mathrm{x}\right\|^{2}$, for all $\mathrm{x} \in \mathrm{H}, \mathrm{y} \in \mathrm{C}$. The metric projection $\mathrm{P}_{\mathrm{C}}$ of $\mathrm{H}$ onto $\mathrm{C}$ satisfies

$<\mathrm{x}-\mathrm{y}, \mathrm{P}_{\mathrm{C}} \mathrm{x}-\mathrm{P}_{\mathrm{C}} \mathrm{y}>\geq\left\|\mathrm{P}_{\mathrm{C}} \mathrm{x}-\mathrm{P}_{\mathrm{C}} \mathrm{y}\right\|^{2}$, for every $\mathrm{x}, \mathrm{y} \in \mathrm{H}$,

Let $\mathrm{A}$ be a monotone mapping of $\mathrm{C}$ into $\mathrm{H}$. In the context of the variational inequality problem, it is easy to see that $\mathrm{u} \in \Omega$ $\Leftrightarrow \mathrm{u}=\mathrm{P}_{\mathrm{C}}(\mathrm{u}-\lambda \mathrm{Au})$, for any $\lambda$

It is known that $\mathrm{H}$ satisfies the Opial condition [7] that is, for any sequence $\left\{\mathrm{x}_{\mathrm{n}}\right\}$ with $\mathrm{x}_{\mathrm{n}} \rightarrow \mathrm{x}$, the inequality, $\lim _{n \rightarrow \infty}$ inf $\| \mathrm{x}_{\mathrm{n}}$ $\mathrm{x} \|<\lim _{n \rightarrow \infty}$ inf $\left\|\mathrm{x}_{\mathrm{n}}-\mathrm{y}\right\|$ holds for every $\mathrm{y} \in \mathrm{H}$ with $\mathrm{y} \neq \mathrm{x}$. We also know that, if $\left\{x_{n}\right\}$ is sequence of $H$ with $x_{n} \rightarrow x$ and $\left\|x_{n}\right\| \rightarrow\|x\|$, then there holds that $x_{n} \rightarrow x$.

A set valued mapping $\mathrm{T}: \mathrm{H} \rightarrow 2^{\mathrm{H}}$ is called monotone if for all $\mathrm{x}, \mathrm{y} \in \mathrm{H}, \mathrm{f} \in \mathrm{Tx}$ and $\mathrm{g} \epsilon$ Ty imply $<\mathrm{x}-\mathrm{y}, \mathrm{f}-\mathrm{g}>\geq 0$. A monotone mapping $\mathrm{T}: \mathrm{H} \rightarrow 2^{\mathrm{H}}$ is maximal if its graph $\mathrm{G}(\mathrm{T})$ is not properly contained in the graph of any other monotone mapping. It is known that a monotone mapping $\mathrm{T}$ is maximal if and only if for $(x, f) \in \mathrm{H} \times \mathrm{H},<\mathrm{x}-\mathrm{y}, \mathrm{f}-\mathrm{g}>\geq 0$ for every $(y, g) \in G(T)$ implies $f \in T x$.

Let $\mathrm{A}: \mathrm{C} \rightarrow \mathrm{H}$ be a monotone, $\mathrm{k}-$ Lipschitz continuous mapping and $\mathrm{N}_{\mathrm{C}} \mathrm{v}$ be the normal cone to $\mathrm{C}$ at $\mathrm{v} \in \mathrm{C}$, that is $\mathrm{N}_{\mathrm{C}} \mathrm{V}$ $=\{\mathrm{w} \in \mathrm{H}:\langle\mathrm{v}-\mathrm{u}, \mathrm{w}>\geq 0, \forall \mathrm{u} \in \mathrm{C}\}$

Define, $\mathrm{T} v= \begin{cases}A v+N_{C} v, & \text { if } v \in C \\ \phi, & \text { if } v \notin C\end{cases}$

Then, $\mathrm{T}$ is maximal monotone [8-9] and $0 € \mathrm{Tv}$ if and only if $\mathrm{V}$ $\epsilon \operatorname{VI}(\mathrm{C}, \mathrm{A})$.

Now we give some lemmas, which will be used in the proof of result.

Lemma 2.1 [6] Let $\mathrm{C}$ be a closed convex subset of a real Hilbert space $\mathrm{H}$. Let $\mathrm{S}$ be a nonexpansive mapping of $\mathrm{C}$ into itself such that $\mathrm{F}(\mathrm{S}) \neq \phi$. Then $\mathrm{F}(\mathrm{S})=\mathrm{F}\left(\mathrm{P}_{\mathrm{C}} \mathrm{S}\right)$.

Lemma 2.2. [12] Let $H$ be a real Hilbert space and let $D$ be a non empty closed convex subset of $H$. Let $\left\{x_{n}\right\}$ be a sequence in $\mathrm{H}$. Suppose that, for all $\mathrm{u} \in \mathrm{D}$,

$\left\|\mathrm{x}_{\mathrm{n}+1}-\mathrm{u}\right\| \leq\left\|\mathrm{x}_{\mathrm{n}}-\mathrm{u}\right\|$,

for every $\mathrm{n}=0,1,2, \ldots \ldots \ldots$. Then, the sequence $\left\{\mathrm{P}_{\mathrm{D}} \mathrm{X}_{\mathrm{n}}\right\}$ converges strongly to some $\mathrm{z} \in \mathrm{D}$.

Lemma 2.3. [3] Let $H$ be a real Hilbert space, $C$ be a nonempty closed convex subset of $\mathrm{H}$ and $\mathrm{T}: \mathrm{C} \rightarrow \mathrm{H}$ be a nonexpansive mapping. Then, the mapping $I-\mathrm{T}$ is demiclosed on C, where $I$ is the identity mapping; that is, $\mathrm{x}_{\mathrm{n}}$ $\rightarrow \mathrm{x}$ in $\mathrm{E}$ and $(I-\mathrm{T}) \mathrm{x}_{\mathrm{n}} \rightarrow \mathrm{y}$ imply that $\mathrm{x} \in \mathrm{C}$ and $(I-\mathrm{T}) \mathrm{x}=$ $\mathrm{y}$.

\section{MAIN RESULT}

Now, we prove a weak convergence theorem for a nonexpansive mapping and a monotone mapping.

Theorem 3.1. Let $\mathrm{C}$ be a closed convex subset of a real Hilbert space $\mathrm{H}$. Let $\mathrm{A}$ be a monotone k-Lipschitz continuous mapping of $\mathrm{C}$ into $\mathrm{H}$ and let $\mathrm{S}$ be a nonexpansive mapping of $\mathrm{C}$ into itself such that $\mathrm{F}(\mathrm{S}) \cap \operatorname{VI}(\mathrm{C}, \mathrm{A}) \neq \phi$. Let $\left\{\mathrm{x}_{\mathrm{n}}\right\}$ be sequence generated by $\mathrm{x}_{0}=\mathrm{x} \in \mathrm{C}$,

$x_{n+1}=P_{C}\left(\alpha_{n} x_{n}+\left(1-\alpha_{n}\right) S P_{C}\left(x_{n}-\lambda_{n} A x_{n}\right)\right)$,

for every $\mathrm{n}=0,1,2, \ldots \ldots \ldots$ where $\left\{\lambda_{n}\right\} \subset[a, b]$ for some $a, b \epsilon$ $(0,2 \alpha)$ and $\left\{\alpha_{n}\right\} \subset[c, d]$ for some $c, d \in(0,1)$. If $\left\{\alpha_{n}\right\}$ and $\left\{\lambda_{n}\right\}$ satisfy the following conditions: $\lim _{n \rightarrow \infty} \alpha_{n}=0, \lim _{n \rightarrow \infty} \lambda_{n}=0, \sum_{n=1}^{\infty}\left|\lambda_{n+1}-\lambda_{n}\right|<\infty$, then, the sequence $\left\{x_{n}\right\}$ converges weakly to some point $\mathrm{z} \epsilon$

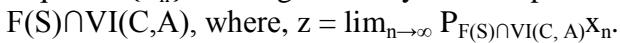

Proof. Let $y_{n}=P_{C}\left(x_{n}-\lambda_{n} A x_{n}\right)$ for every $n=0,1,2,3, \ldots$

Let $u \in F(S) \cap \operatorname{VI}(C, A)$.

Now,

$\left\|\mathrm{y}_{\mathrm{n}}-\mathrm{u}\right\|^{2} \leq\left\|\mathrm{x}_{\mathrm{n}}-\lambda_{\mathrm{n}} A \mathrm{x}_{\mathrm{n}}-\mathrm{u}\right\|^{2}-\left\|\mathrm{x}_{\mathrm{n}}-\lambda_{\mathrm{n}} A \mathrm{x}_{\mathrm{n}}-\mathrm{y}_{\mathrm{n}}\right\|^{2}$

$=\left\|x_{n}-u\right\|^{2}+\left\|\lambda_{n} A x_{n}\right\|^{2}-2 \lambda_{n}<x_{n}-u, A x_{n}>-\left\|x_{n}-y_{n}\right\|^{2}-\|$

$\lambda_{\mathrm{n}} A \mathrm{x}_{\mathrm{n}} \|+2 \lambda_{\mathrm{n}}<\mathrm{x}_{\mathrm{n}}-\mathrm{y}_{\mathrm{n}}, A \mathrm{x}_{\mathrm{n}}>$

$=\left\|x_{n}-u\right\|^{2}-\left\|x_{n}-y_{n}\right\|^{2}+2 \lambda_{n}\left\langle x_{n}-y_{n}-x_{n}+u, A x_{n}>\right.$

$=\left\|x_{n}-u\right\|^{2}-\left\|x_{n}-y_{n}\right\|^{2}+2 \lambda_{n}<A x_{n}, u-y_{n}>$

$=\left\|x_{n}-u\right\|^{2}-\left\|x_{n}-y_{n}\right\|^{2}+2 \lambda_{n}\left(<A x_{n}-A u, u-x_{n}>+<A u, u-\right.$

$\left.\mathrm{x}_{\mathrm{n}}>+<A x_{\mathrm{n}}, \mathrm{x}_{\mathrm{n}}-\mathrm{y}_{\mathrm{n}}>\right)$

$\leq\left\|x_{n}-u\right\|^{2}-\left\|x_{n}-y_{n}\right\|^{2}+2<\lambda_{n} A x_{n}, x_{n}-y_{n}>$

$\left.=\left\|x_{n}-u\right\|^{2}-\left\|x_{n}-y_{n}\right\|^{2}+2<x_{n}+\lambda_{n} A x_{n}-y_{n}, x_{n}-y_{n}\right\rangle+<y_{n}-$

$\mathrm{x}_{\mathrm{n},} \mathrm{x}_{\mathrm{n}}-\mathrm{y}_{\mathrm{n}}>$

$\leq\left\|x_{n}-u\right\|^{2}-\left\|x_{n}-y_{n}\right\|^{2}-\left\|x_{n}-y_{n}\right\|^{2}$

$=\left\|\mathrm{x}_{\mathrm{n}}-\mathrm{u}\right\|^{2}-2\left\|\mathrm{x}_{\mathrm{n}}-\mathrm{y}_{\mathrm{n}}\right\|^{2}$

$\leq\left\|\mathrm{x}_{\mathrm{n}} \mathrm{-u}\right\|^{2}$ for every $\mathrm{n}=0,1,2, \ldots$.

Now, using (1), we obtain,

$\left\|\mathrm{x}_{\mathrm{n}+1}-\mathrm{u}\right\|^{2}$

$=\left\|\mathrm{P}_{\mathrm{C}}\left(\alpha_{\mathrm{n}} \mathrm{x}_{\mathrm{n}}+\left(1-\alpha_{\mathrm{n}}\right) S \mathrm{y}_{\mathrm{n}}\right)-\mathrm{P}_{\mathrm{C}} \mathrm{Su}\right\|^{2}$

$\leq\left\|\left(\alpha_{n} x_{n}+\left(1-\alpha_{n}\right) S y_{n}\right)-S u\right\|^{2}$

$=\left\|\alpha_{n}\left(x_{n}-u\right)+\left(1-\alpha_{n}\right)\left(S y_{n}-S u\right)\right\|^{2}$

$=\left\|\alpha_{n}\left(x_{n}-u\right)+\left(1-\alpha_{n}\right)\left(y_{n}-u\right)\right\|^{2}$

$\leq \alpha_{n}\left\|x_{n}-u\right\|^{2}+\left(1-\alpha_{n}\right)\left\|y_{n}-u\right\|^{2}$

$\leq \alpha_{n}\left\|x_{n}-u\right\|^{2}+\left(1-\alpha_{n}\right)\left(\left\|x_{n}-u\right\|^{2}-2\left\|x_{n}-y_{n}\right\|^{2}\right)$

$\leq\left\|x_{n}-u\right\|^{2}-2\left(1-\alpha_{n}\right)\left\|x_{n}-y_{n}\right\|^{2}$

$\leq\left\|\mathrm{x}_{\mathrm{n}}-\mathrm{u}\right\|^{2}$

So, $\left\|x_{n+1}-u\right\| \leq\left\|x_{n}-u\right\|$

So, there exists $c=\lim _{n \rightarrow \infty}\left\|x_{n}-u\right\|$ and hence the sequences $\left\{x_{n}\right\},\left\{y_{n}\right\}$ are bounded. From equation (2),

$2\left(1-\alpha_{n}\right)\left\|x_{n}-y_{n}\right\|^{2} \leq\left\|x_{n}-u\right\|^{2}-\left\|x_{n+1}-u\right\|^{2}$

Since, $\lim _{n \rightarrow \infty}\left\|x_{n}-u\right\|^{2}=\lim _{n \rightarrow \infty}\left\|x_{n+1}-u\right\|^{2}$

So we obtain, $x_{n}-y_{n} \rightarrow 0$.

Since A is lipschitz continuous, so $\mathrm{Ax}_{\mathrm{n}}-\mathrm{Ay}_{\mathrm{n}} \rightarrow 0$.

Now,

$\left\|\mathrm{y}_{\mathrm{n}}-\mathrm{y}_{\mathrm{n}-1}\right\| \leq\left\|\mathrm{P}_{\mathrm{C}}\left(\mathrm{x}_{\mathrm{n}}-\lambda_{\mathrm{n}} \mathrm{Ax}_{\mathrm{n}}\right)-\mathrm{P}_{\mathrm{C}}\left(\mathrm{x}_{\mathrm{n}-1}-\lambda_{\mathrm{n}-1} \mathrm{Ax}_{\mathrm{n}-1}\right)\right\|$

$\leq\left\|\left(x_{n}-\lambda_{n} A x_{n}\right)-\left(x_{n-1}-\lambda_{n-1} A x_{n-1}\right)\right\|$

$=\left\|\left(\mathrm{x}_{\mathrm{n}}-\mathrm{x}_{\mathrm{n}-1}\right)-\left(\lambda_{\mathrm{n}} \mathrm{Ax} \mathrm{x}_{\mathrm{n}}-\lambda_{\mathrm{n}-1} \mathrm{Ax}_{\mathrm{n}-1}\right)\right\|$

$=\left\|\left(x_{n}-x_{n-1}\right)-\left(\lambda_{n} A x_{n}-\lambda_{n} A x_{n-1}+\lambda_{n} A x_{n-1}-\lambda_{n-1} A x_{n-1}\right)\right\|$

$\leq\left\|\left(\mathrm{x}_{\mathrm{n}}-\mathrm{x}_{\mathrm{n}-1}\right)\right\|+\lambda_{\mathrm{n}}\left\|\mathrm{Ax}_{\mathrm{n}}-\mathrm{Ax}_{\mathrm{n}-1}\right\|+\left|\lambda_{\mathrm{n}}-\lambda_{\mathrm{n}-1}\right|\left\|\mathrm{Ax}_{\mathrm{n}-1}\right\|$

$\leq\left(1+\lambda_{\mathrm{n}} \mathrm{k}\right)\left\|\left(\mathrm{x}_{\mathrm{n}}-\mathrm{x}_{\mathrm{n}-1}\right)\right\|+\left|\lambda_{\mathrm{n}}-\lambda_{\mathrm{n}-1}\right|\left\|\mathrm{Ax}_{\mathrm{n}-1}\right\|$ 
Using the given conditions, we obtain,

$\left\|\mathrm{y}_{\mathrm{n}}-\mathrm{y}_{\mathrm{n}-1}\right\| \leq\left\|\left(\mathrm{x}_{\mathrm{n}}-\mathrm{x}_{\mathrm{n}-1}\right)\right\|$.

Since,

$\left\|\mathrm{x}_{\mathrm{n}+1}-\mathrm{x}_{\mathrm{n}}\right\| \leq\left\|\mathrm{x}_{\mathrm{n}+1}-\mathrm{y}_{\mathrm{n}}\right\|+\left\|\mathrm{y}_{\mathrm{n}}-\mathrm{x}_{\mathrm{n}}\right\| \rightarrow 0$ as $\mathrm{n} \rightarrow \infty$, so (3) implies,

$\left\|\mathrm{y}_{\mathrm{n}}-\mathrm{y}_{\mathrm{n}-1}\right\| \rightarrow 0$ as $\mathrm{n} \rightarrow \infty$.

Now, using (4) and given conditions:

$\left\|\mathrm{x}_{\mathrm{n}}-\mathrm{P}_{\mathrm{C}} S \mathrm{y}_{\mathrm{n}}\right\| \leq\left\|\mathrm{x}_{\mathrm{n}}-\mathrm{P}_{\mathrm{C}} S \mathrm{y}_{\mathrm{n}-1}\right\|+\left\|\mathrm{P}_{\mathrm{C}} S \mathrm{y}_{\mathrm{n}-1}-\mathrm{P}_{\mathrm{C}} S \mathrm{y}_{\mathrm{n}}\right\|$

$\leq \alpha_{\mathrm{n}-1}\left\|\mathrm{x}_{\mathrm{n}-1}-\mathrm{Sy}_{\mathrm{n}-1}\right\|+\left\|\mathrm{y}_{\mathrm{n}-1}-\mathrm{y}_{\mathrm{n}}\right\| \rightarrow 0$ as $\mathrm{n} \rightarrow \infty$.

Also, $\left\|\mathrm{y}_{\mathrm{n}}-\mathrm{P}_{\mathrm{C}} \mathrm{Sy}_{\mathrm{n}}\right\| \leq\left\|\mathrm{x}_{\mathrm{n}}-\mathrm{P}_{\mathrm{C}} \mathrm{Sy}_{\mathrm{n}}\right\|+\left\|\mathrm{y}_{\mathrm{n}}-\mathrm{x}_{\mathrm{n}}\right\| \rightarrow 0$ as $\mathrm{n} \rightarrow$ $\infty$.

As $\left\{x_{n}\right\}$ is bounded, we have that a subsequence $\left\{x_{n_{i}}\right\}$ of $\left\{x_{n}\right\}$ that converges weakly to $z$. Then, we shall prove that $\mathrm{z} \in$ $\mathrm{F}(\mathrm{S}) \cap \mathrm{VI}(\mathrm{C}, \mathrm{A})$.

Firstly, we shall show that $\mathrm{z} \in \mathrm{VI}(\mathrm{C}, \mathrm{A})$.

Since $\mathrm{x}_{\mathrm{n}}-\mathrm{y}_{\mathrm{n}} \rightarrow 0$, we have, $y_{n_{i}} \rightarrow \mathrm{z}$. Let

$\mathrm{T} v= \begin{cases}A v+N_{C} v, & \text { if } v \in C \\ \phi, & \text { if } v \notin C\end{cases}$

Then $T$ is maximal monotone. Let $(v, w) \in G(T)$. Since $w$ $A v \in N_{C} v$ and $y_{n} \in C$, so we get

$\left\langle\mathrm{v}-\mathrm{y}_{\mathrm{n}, \mathrm{w}}-\mathrm{Av}\right\rangle \geq 0$.

On the other hand, from $y_{n}=P_{C}\left(x_{n}-\lambda_{n} A x_{n}\right)$, we have that

$<\mathrm{x}_{\mathrm{n}}-\lambda_{\mathrm{n}} \mathrm{Ax}_{\mathrm{n}}-\mathrm{y}_{\mathrm{n},} \mathrm{y}_{\mathrm{n}}-\mathrm{v}>\geq 0$ and hence, $<\mathrm{v}-\mathrm{y}_{\mathrm{n}},\left(\mathrm{y}_{\mathrm{n}}-\mathrm{x}_{\mathrm{n}}\right) / \lambda_{\mathrm{n}}+$ $A x_{n}>\geq 0$.

Therefore, we have

$<\mathrm{v}-y_{n_{i}}, \mathrm{w}>\geq<\mathrm{v}-y_{n_{i}}, \mathrm{Av}>$

$\geq<\mathrm{v}-y_{n_{i}}, \mathrm{Av}>-<\mathrm{v}-y_{n_{i}},\left(y_{n_{i}}-x_{n_{i}}\right) / \lambda_{n_{i}}+\mathrm{A} x_{n_{i}}>$

$=\left\langle\mathrm{v}-y_{n_{i}}, \operatorname{Av}-\mathrm{A} x_{n_{i}}-\left(y_{n_{i}}-x_{n_{i}}\right) / \lambda_{n_{i}}>\right.$

$=<\mathrm{v}-y_{n_{i}}, \mathrm{Av}-\mathrm{A} y_{n_{i}}>+<\mathrm{v}-y_{n_{i}}, \mathrm{~A} y_{n_{i}}-\mathrm{A} x_{n_{i}}>-<\mathrm{v}-$

$y_{n_{i}},\left(y_{n_{i}}-x_{n_{i}}\right) / \lambda_{n_{i}}>$

$\geq<\mathrm{v}-y_{n_{i}}, \mathrm{~A} y_{n_{i}}-\mathrm{A} x_{n_{i}}>-<\mathrm{v}-y_{n_{i}},\left(y_{n_{i}}-x_{n_{i}}\right) / \lambda_{n_{i}}>$.

Hence we get, $\langle\mathrm{v}-\mathrm{z}, \mathrm{w}>\geq 0, \quad$ as $\mathrm{i} \rightarrow \infty$.

Since $\mathrm{T}$ is maximal monotone, we have $\mathrm{z} \epsilon \mathrm{T}^{-1} 0$ and hence $\mathrm{z} \epsilon$ $\mathrm{VI}(\mathrm{C}, \mathrm{A})$.

Next we shall show that $z \in F\left(P_{C} S\right)$.

Since $y_{n_{i}}-\mathrm{z}$ and $\left\|\mathrm{y}_{\mathrm{n}}-\mathrm{P}_{\mathrm{C}} \mathrm{Sy}_{\mathrm{n}}\right\| \rightarrow 0$ as $\mathrm{n} \rightarrow \infty$, so by demiclosedness of $\mathrm{I}-\mathrm{S}$, we get $\mathrm{z} \in \mathrm{F}\left(\mathrm{P}_{\mathrm{C}} \mathrm{S}\right)$.
By using Lemma 2.1, we obtain $\mathrm{z} \in \mathrm{F}(\mathrm{S})$.

Let $\left\{X_{n_{j}}\right\}$ be another subsequence of $\left\{x_{n}\right\}$, such that $\left\{X_{n_{j}}\right.$ \}$\rightarrow z^{\prime}$. Then, $z^{\prime} \epsilon \mathrm{F}(\mathrm{S}) \cap \mathrm{VI}(\mathrm{C}, \mathrm{A})$. Let us show that $\mathrm{z}=\mathrm{z}$. Assume that $\mathrm{z} \neq \mathrm{z}$. From the Opial condition, we have

$\lim _{n \rightarrow \infty}\left\|x_{n}-z\right\|$

$=\lim _{i \rightarrow \infty} \inf \left\|x_{n_{i}}-z\right\|$

$<\lim _{i \rightarrow \infty}$ inf $\left\|x_{n_{i}}-z^{\prime}\right\|$

$=\lim _{\mathrm{n} \rightarrow \infty}\left\|\mathrm{x}_{\mathrm{n}}-\mathrm{z}^{\prime}\right\|=\lim _{j \rightarrow \infty}$ inf $\left\|x_{n_{j}}-\mathrm{z}^{\prime}\right\|<\lim _{j \rightarrow \infty}$ inf $\|$

$x_{n_{j}}-\mathrm{z}\left\|=\lim _{\mathrm{n} \rightarrow \infty}\right\| \mathrm{x}_{\mathrm{n}}-\mathrm{z} \|$.

This is a contradiction. Thus we have $z=z^{\prime}$. This implies, $\mathrm{x}_{\mathrm{n}} \rightarrow \mathrm{z} \in \mathrm{F}(\mathrm{S}) \cap \mathrm{VI}(\mathrm{C}, \mathrm{A})$.

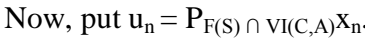

We show that $\mathrm{z}=\lim _{\mathrm{n} \rightarrow \infty} \mathrm{u}_{\mathrm{n}}$.

From, $\mathrm{u}_{\mathrm{n}}=\mathrm{P}_{\mathrm{F}(\mathrm{S}) \cap \mathrm{VI}(\mathrm{C}, \mathrm{A})} \mathrm{x}_{\mathrm{n}}$ and $\mathrm{z} \in \mathrm{F}(\mathrm{S}) \cap \mathrm{VI}(\mathrm{C}, \mathrm{A})$, we have, $<\mathrm{z}$ $-u_{n}, u_{n}-x_{n}>\geq 0$.

By lemma 2.2, $\left\{\mathrm{u}_{\mathrm{n}}\right\}$ converges strongly to some $\mathrm{z}_{0} \in \mathrm{F}(\mathrm{S}) \cap$ $\mathrm{VI}(\mathrm{C}, \mathrm{A})$.

Then, we have, $<\mathrm{z}-\mathrm{z}_{0}, \mathrm{z}_{0}-\mathrm{z}>\geq 0$.

And hence $\mathrm{z}=\mathrm{z}_{0}$.

Remark. We know that every inverse-strongly-monotone mapping is monotone and Lipschitz continuous, but converse is not true. So our result is the generalization of Theorem 1.1 given by Takahashi and Toyoda [12] and Theorem 1.2 given by Iiduka and Takahashi [4].

\section{NUMERICAL EXAMPLE}

Now we prove the validity of our main result with the help of a numerical example and a program in $\mathrm{C}++$.

Example 4.1. Let $H=R$ be a real Hilbert space with usual inner product defined on $\mathrm{R}$. Let $\mathrm{C}=[0,1]$ be a closed convex subset of $\mathrm{H}$. Let $\mathrm{Ax}=\frac{x^{2}}{1+x}$ be a monotone, Lipschitzcontinuous mapping of $\mathrm{C}$ into $\mathrm{H}$ and let $\mathrm{Sx}=\frac{x}{2}$ be a nonexpansive mapping of $\mathrm{C}$ into itself. Let $\left\{\alpha_{n}\right\}=\left\{\lambda_{n}\right\}=$ $\frac{1}{n+1}$ be two sequences satisfying the conditions of theorem 3.1 .

Now using the iterative scheme (3.1.1), we obtain the following data given in table 4.1. when initial approximation is taken as $\mathrm{x}_{0}=0.1$. 
Table 4.1

\begin{tabular}{|l|l|l|l|}
\hline $\mathrm{n}$ & $\mathrm{x}_{\mathrm{n}+1}$ & $\mathrm{Sx}_{\mathrm{n}}$ & $\mathrm{P}_{\mathrm{C}}\left(\mathrm{x}_{\mathrm{n}}-\lambda_{\mathrm{n}} \mathrm{Ax} \mathrm{x}_{\mathrm{n}}\right)$ \\
\hline 0 & 0.1 & 0.05 & 0.1 \\
\hline 1 & 0.045455 & 0.045455 & 0.090909 \\
\hline 3 & 0.022727 & 0.022727 & 0.045455 \\
\hline 98 & $2.868585 \mathrm{e}-31$ & $2.868585 \mathrm{e}-31$ & $5.73717 \mathrm{e}-31$ \\
\hline 99 & $1.434293 \mathrm{e}-31$ & $1.434293 \mathrm{e}-31$ & $2.868585 \mathrm{e}-31$ \\
\hline 100 & $7.171463 \mathrm{e}-32$ & $7.171463 \mathrm{e}-32$ & $1.434293 \mathrm{e}-31$ \\
\hline 279 & $9.359196 \mathrm{e}-86$ & $9.359196 \mathrm{e}-86$ & $1.871839 \mathrm{e}-85$ \\
\hline 280 & $4.679598 \mathrm{e}-86$ & $4.679598 \mathrm{e}-86$ & $9.359196 \mathrm{e}-86$ \\
\hline 770 & $1.463897 \mathrm{e}-233$ & $1.463897 \mathrm{e}-233$ & $2.927795 \mathrm{e}-233$ \\
\hline 771 & $7.319487 \mathrm{e}-234$ & $7.319487 \mathrm{e}-234$ & $1.463897 \mathrm{e}-233$ \\
\hline 1071 & $4.940656 \mathrm{e}-324$ & $4.940656 \mathrm{e}-324$ & $9.881313 \mathrm{e}-324$ \\
\hline 1072 & 0 & 0 & $4.940656 \mathrm{e}-324$ \\
\hline 1073 & 0 & 0 & 0 \\
\hline 1074 & 0 & 0 & 0 \\
\hline
\end{tabular}

Thus we see that the sequence $\left\{x_{n}\right\}$ converges to $z=0$, which is a fixed point of $\mathrm{S}$ as well as a solution of variational inequality problem.

\section{APPLICATION}

Theorem: Let $\mathrm{H}$ be a real Hilbert space. Let $\mathrm{A}$ be a monotone $\mathrm{k}$-Lipschitz continuous mapping of $\mathrm{H}$ into itself and let $\mathrm{S}$ be a nonexpansive mapping of $\mathrm{H}$ into itself such that $\mathrm{F}(\mathrm{S}) \cap \mathrm{A}^{-1} 0$ $\neq \phi$. Let $\left\{\mathrm{x}_{\mathrm{n}}\right\}$ be a sequence generated by $\mathrm{x}_{0}=\mathrm{x} \in \mathrm{H}$ and let

$x_{n+1}=P_{C}\left(\alpha_{n} x_{n}+\left(1-\alpha_{n}\right) S\left(x_{n}-\lambda_{n} A x_{n}\right)\right)$,

for every $\mathrm{n}=0,1,2, \ldots \ldots \ldots$ where $\left\{\lambda_{\mathrm{n}}\right\} \subset[\mathrm{a}, \mathrm{b}]$ for some $\mathrm{a}, \mathrm{b} \epsilon$ $(0,1 / k)$ and $\left\{\alpha_{n}\right\} \subset[c, d]$ for some $c, d \in(0,1)$. Then, the sequence $\left\{x_{n}\right\}$ converges weakly to some point $z \in F(S) \cap A^{-}$

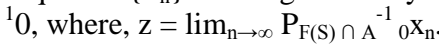

Proof. We have $\mathrm{A}^{-1} 0=\mathrm{VI}(\mathrm{H}, \mathrm{A})$ and $\mathrm{P}_{\mathrm{H}}=\mathrm{I}$. By Theorem 3.1, we obtain the desired result.

Remark. Notice that $\mathrm{F}(\mathrm{S}) \cap \mathrm{A}^{-1} 0 \subset \mathrm{VI}(\mathrm{F}(\mathrm{S}), \mathrm{A})$. See Yamada [13] for the case when A is strongly monotone and Lipschitz continuous mapping of $\mathrm{H}$ into itself.

\section{REFERENCES}

[1] Browder F E and Petryshyn W V (1967) Construction of Fixed Points of non-linear Mapping in Hilbert Spaces J Math Anal Appl 20 197-228.

[2] Browder F E (1965) Fixed-point Theorems for Noncompact Mappings in Hilbert Space Proc Natl Acad Sci U S A 53 1272-1276.

[3] Goebel K and Kirk W A (1990) Topics in Metric Fixed Point Theory vol. 28 of Cambridge Studies in Advanced Mathematics, Cambridge University Press, Cambridge, UK.

[4] Iiduka H and Takahashi W (2004), Stronge convergence theorems for nonexpansive nonself mappings and inverse strongly monotone mappings, J convex Anal, vol 11, 69 79 .

[5] Liu F and Nashed M Z (1998) Regularization of Nonlinear Ill- posed Variational inequalities and convergence rates,Set-valued Anal 6 313-344.

[6] Matsushita S and Kuroiwa, Approximation of fixed points of nonexpansive nonself mappings, Sci Math Jpn, vol 57(2003), 171-176.

[7] Opial Z (1967) Weak convergence of the sequence of successive Approximations of Nonexpansive Mappings Bull Am Math Soc 73 591-597.

[8] Rockafellar R T (1970) On the Maximality of Sums of nonlinear Monotone Operators Trans Amer Math Soc 149 75-88.

[9] Schu J (1991) Weak and Strong Convergence to fixed points of Asymptotically nonexpansive Mappings Bull Aust Math Soc 43, 153-159.

[10] Takahashi W (2000) Non-linear functional Analysis Yokohama Publisher, Yokohama Japan.

[11] Takahashi W, Tamura T (1998) Convergence Theorems for a pair of nonexpansive Mapping J Convex Anal 5 4556.

[12] Takahashi W and Toyoda M (2003) Weak Convergence Theorems for Non-expansive Mappings and Monotone Mappings J Optim Theory Appl 118 417-428.

[13] Yamda I (2001) The Hybrid Steepest-Descent Method for the Variational Inequality Problem over the Intersection of fixed-point Sets of nonexpansive Mapping, inherently Parallel Algorithms in Feasibility and Optimization and their applications, Edited by D Butnariu, y. Censor, and S. Reich, kluwer Academic Pulishers, Dordrecht, Holland, 473-504. 